\title{
The decoherence criterion
}

\author{
Eleonora Alfinito ${ }^{a}$ ], Rosario G. Viglione ${ }^{b}$ Z $^{2}$ and Giuseppe Vitiello ${ }^{a}$ ] \\ ${ }^{a}$ Dipartimento di Fisica, Università di Salerno, 84100 \\ and INFN Gruppo Collegato di Salerno and INFM Unità di Salerno \\ ${ }^{b}$ Dipartimento di Chimica, Università di Salerno, 84100
}

\begin{abstract}
The decoherence mechanism signals the limits beyond which the system dynamics approaches the classical behavior. We show that in some cases decoherence may also signal the limits beyond which the system dynamics has to be described by quantum field theory, rather than by quantum mechanics.
\end{abstract}

The problem of the range of applicability of quantum mechanics (QM) has been the object of much attention and studies since the early days of its foundation. However, only at a later time it has been fully recognized and appreciated the purely quantum phenomenon of decoherence [1, 2, 3, 4, 5], which in many cases signals the appearance of a classical world in quantum theory [5]. Decoherence is normally triggered by the interaction of the system with the environment and formally consists in suppressing the off-diagonal elements of the reduced density matrix. Its effect is such that quantum superposition in the system wave function is destroyed and thus, provided the time-scale $\tau_{d y n}$ characteristic of the dynamics is much greater than the decoherence time-scale $\tau_{d e c}, \tau_{d y n} \gg \tau_{d e c}$, the classical behavior may be approached. It should be noted that sometimes the system under consideration, assumed to be a closed system, is viewed as composed of two subsystems and we are actually interested only in one of them. The other subsystem is then regarded to be the environment in which the former one is embedded. The reduced density matrix is the one obtained by tracing over the degrees of freedom of the environment (the rest of the Universe, whatever it is).

\footnotetext{
1 alfinito@sa.infn.it

2 ros@alex.chem.unisa.it

${ }^{3}$ vitiello@sa.infn.it
} 
Decoherence provides an interesting explanation why we do not experience superpositions of objects in the macroscopic world. In fact interaction with the environment produces decoherence in the superposition of macroscopically separate positions so that the familiar classical behavior of non-superposing macroscopic objects is obtained. However, we also have experience (and sound theoretical understanding) of the existence of macroscopic quantum systems, such as superconductors, ferromagnets, crystals, and in general systems presenting ordered patterns, where coherence over macroscopically large distances appears to be particularly stable against environment perturbations. Any system is made by quantum components. It is, of course, not in such a trivial sense that macroscopic systems such as a superconductor or a crystal are quantum systems. These are quantum systems in the specific, non-trivial sense that their macroscopic (classical) behavior cannot be explained without recourse to quantum theory. It is then natural, and necessary, asking the question of the consistency between the decoherence phenomenon and the existence of macroscopic quantum systems. This is indeed the question we address in this paper.

Our conclusion will be that the decoherence mechanism characterizes QM by designing its borderlines also with quantum field theory (QFT), besides, as already known, with classical mechanics. In other words, our result suggests the use of the decoherence mechanism as an useful criterion to scan the border QM/QFT.

For the sake of clarity it might be convenient to start our discussion by shortly reviewing some general aspects of the QFT approach to the study of macroscopic quantum systems, such as superconductors, ferromagnets, crystals, etc. These systems have the common feature of presenting some kind of ordered patterns in the ground state. The observable describing such an ordering is called the order parameter and it is a macroscopic observable in the sense that it is not strongly affected by quantum fluctuations. The spontaneous breakdown of symmetry in QFT provides the mechanism out of which the ordered patterns are dynamically generated.

Suppose that the system Lagrangian (or the field equations from it derived) is invariant under some group $\mathrm{G}$ of symmetry transformations. The symmetry is said to be spontaneously broken when the lowest energy state (the vacuum or ground state) of the physical system is not invariant under the same symmetry group G, but under one of its subgroups. For example, for a metal, which below some critical temperature may exhibit superconductivity, the Lagrangian is invariant under the $\mathrm{U}(1)$ transformations. However, the ground state of the metal in the superconducting phase is not invariant under the $\mathrm{U}(1)$ transformations. The ground state of the same metal above the critical temperature, in the "normal" (i.e. non superconducting) phase, is, on the contrary, U(1) symmetric. The Lagrangian for a gas of particles endowed with magnetic moment, which under specific boundary conditions may be found in the ferromagnetic phase, is invariant under the $\mathrm{SU}(2)$ group of rotations. However, the system in the ferromagnetic phase, below the Curie temperature, is invariant under the $\mathrm{U}(1)$ subgroup of the original $\mathrm{SU}(2)$. The Lagrangian for a gas of atoms or molecules, which below a critical temperature may be arranged in a crystal phase, is invariant under the continuous space translations. However, the 
same system of atoms or molecules when it is in the crystal phase has the lowest energy state which is not invariant under continuous space translations, but under discrete translations of the length of the lattice spacing or of its multiples. Whenever, the symmetry of the Lagrangian is not the same as the symmetry of the vacuum we have spontaneous symmetry breakdown. The dynamics of a system, given in terms of the Lagrangian, may thus generate at the observable level, under different boundary conditions (e.g. different temperatures), different phases of the system (the normal phase and the superconducting phase, the ferromagnetic phase and the non-ferromagnetic one, the crystal phase and the amorphous one). The crystal ordering, the superconductor ordering, etc., thus appears in the non-symmetric (or "less" symmetric) ground state: order is in fact lack of symmetry.

The Goldstone theorem [6] states that whenever a continuous symmetry is spontaneously broken the dynamics implies the existence of gapless (massless) modes: they are called the Nambu-Goldstone (NG) boson particles or quanta. We will not analyze the case in which there is a gauge field in the theory, which would not change the substance of our conclusions. Also, we will not report the derivation of the Goldstone theorem since it can be easily found in any standard textbook of QFT (see e.g. [6]).

The Nambu-Goldstone bosons are the quanta of long range correlation among the system constituents. Examples of NG boson quanta are the phonons in the crystals, the magnons in the ferromagnets, the Cooper pairs in the superconductors. As already said, these quanta are of dynamical origin, namely they are not found in the symmetric or normal phases (which are typically obtained above some critical temperature). The NG bosons are thus collective modes, the long range correlation, of which they are the quanta, is responsible for the ordering in the ground state of the non-symmetric (ordered) phase: the NG quanta are the carriers of the ordering information throughout the system. It is in this way that the order is generated by the quantum dynamics and appears as a macroscopic, diffused property of the system in the non-symmetric phase. In other words, in the ordered phase the system components get, so to say, "trapped" by the long range correlation, they cannot behave as individual particles. Some of their degrees of freedom get frozen by the NG long range correlation, which is a dynamical consequence of the "lack" of symmetry, and this manifests itself as the system macroscopically observable ordered patterns. We thus arrive at the understanding of the existence of macroscopic quantum systems. All of this is formally expressed in terms of coherent condensation of NG boson quanta in the ground state [6, 7, 8, 9]. Since these quanta are massless their coherent condensation in the lowest energy mode does not add energy to the ground state and the observed high stability of the ordered phases is thus explained. The mathematical formalism of the many-body theory of superconductors, ferromagnets, crystals and other macroscopic quantum systems is given in great details in standard textbooks [6, 7, 8] and therefore we do not report it here.

A given system thus may possesses degenerate ground states, each corresponding to a physically different phase in which the system may be observed under different boundary conditions. These degenerate ground states are in fact unitarily inequiv- 
alent, i.e. it does not exist any unitary operator transforming one of them into another one. In different words, one cannot express the ground state of a specific phase in terms of the ground state of another, different phase: the crystal ground state cannot be expressed in terms of the amorphous ground state, the superconducting ground state cannot be expressed in terms of the normal one, and so on. As well known [6] - [12], this is formally expressed, e.g. for the superconducting Bogoliubov or $B C S$ ground state $\mid 0(B C S)>$, by

$$
<0 \mid 0(B C S)>\rightarrow 0 \quad \text { for } \quad V \rightarrow \infty,
$$

in the infinite number of degrees of freedom limit (infinite volume $V$ limit). Here $\mid 0>$ denotes the "normal" ground state (i.e. the non-superconducting phase ground state), and $\mid 0(B C S)>$ is formally expressed at finite volume as $|0(B C S)\rangle \equiv$ $\Pi_{k}\left(U_{k}+V_{k} c_{\mathbf{k}+}^{\dagger} c_{-\mathbf{k}-}^{\dagger}\right) \mid 0>$, with $U_{k}^{2}+V_{k}^{2}=1$ and $c_{\mathbf{k}+}^{\dagger}$ denoting the creation operators for the spin up and down electron, respectively [14]. The meaning of Eq. (11) is that in the infinite volume limit the ordered ground state $\mid 0(B C S)>$ cannot be reached by building up perturbations around the non-ordered one $\mid 0>$ : the state $\mid 0(B C S)>$ cannot be expressed in terms of $\mid 0>$, and vice-versa. We have a typical non-perturbative phenomenon: the different physical phases are described by states belonging respectively to different Hilbert spaces, each of them corresponding to a specific ground state. These physically different spaces are the unitarily inequivalent representations of the canonical commutation relations and, for the above example, we denote them by $\{\mid 0>\}$ and $\{\mid 0(B C S)>\}$. Eq. (1) is a typical QFT result: in fact (11), which states the unitary inequivalence between the representations $\{\mid 0>\}$ and $\{\mid 0(B C S)>\}$, cannot hold in QM where the von Neumann theorem states that all the representations are unitarily, and thus physically, equivalent [10, 11, 12, 13]. On the contrary, physically different phases are allowed in QFT, since the von Neumann theorem does not hold in QFT and there exist infinitely many unitarily inequivalent representations of the canonical commutation relations.

Eq. (1) thus states that the systems presenting different physical phases cannot be described purely in terms of QM. QM is not enough "rich" to allow unitarily inequivalent representations, as rigorously implied by the von Neuman theorem. In this paper we are going indeed to confirm such a result from the perspective of the decoherence mechanism in QM.

We will consider as an example the commonly observed process of the formation of a crystal out of an ion solution. The result implied by QM decoherence formulas would prevent the formation of the crystal, since the computed decoherence time for the ions in the solution is too much short with respect to the time one has to wait before the crystal gets formed in common observations. This does not mean that those QM formulas are wrong, neither, of course, it means that our system is a classical one. It means that it is wrong to apply these QM formulas to the ions in solution in order to study the process of the crystal formation. Thus, in QM, once one starts with the ion solution phase, dechoerence tells us that no other phase (the crystal phase) is reachable, which indeed is in perfect agreement with what the von Neumann theorem states. 
Let us now turn our attention to the QM decoherence scenario. There is a large body of literature on decoherence and for the sake of shortness we do not reproduce here the already published derivations of the formulas we are going to use. We refer for that to the quoted papers. To be specific, we focus on the formation of, e.g., the binary crystals listed in Table 1 . We stress that the system we study is not the already formed crystal, but the solution of ions out of which one expects (observes) the crystal will be formed. Thus, according to the usual chemical recipe, we consider a solution (typically, the water is the solvent) of the constituent elements (e.g. a solution of $\mathrm{Na}^{+}$ions and $\mathrm{Cl}^{-}$ions) and wait, in specific conditions of temperature, density, etc., till the crystallization occurs. This happens when the saturation of the solution is reached. At the crystallization point, the saturation concentrations can be quite different in different cases, depending on the crystal one wants to obtain, ranging, for example, from 1 ion of $K^{+}$for 4 molecules of water for $K F$, or 1 ion of $\mathrm{Na}^{+}$for 10 molecules of water for $\mathrm{NaCl}$, to 1 ion of $\mathrm{Ag}^{+}$for $10^{8}$ molecules of water for $\mathrm{AgBr}$, till 1 ion of $\mathrm{Pb}^{+}$for $10^{15}$ molecules of water for $\mathrm{PbS}$ 15. The ions in the solution are normally bound, due to Coulomb forces, to water molecules, for example the $\mathrm{Na}^{+}$ion is surrounded by four water molecules. The shielding of the ionic charge by the surrounding water molecules lowers the intensity of the Coulomb interaction among ions. Sometimes one adds a "germ", namely a small crystal of the same kind of the one to be formed. Such a germ will act as a catalytic structure making more favorable the aggregation, in the wanted crystal structure, of the ions in the solution. Sometimes the nucleation is simply produced by some "defect" or "impurity", e.g., on the walls of the bowl or container of the solution. One observes the crystal formation in the vicinity of these defects. At the crystallization point, lowering the temperature of the solution normally helps the crystal formation, which can occur within a short lapse of time (from fractions of a second to several seconds) or in a longer one (from minutes to hours).

The interaction among the ions in the solution is of Coulomb type and we will consider the ion-ion collisions and the interactions with distant ions to be two possible sources of decoherence. There are also other sources of decoherence such as, e.g., the interaction with the environment (the water in our case), with the crystal germ or with the defects or impurities, or else with dipole and higher moments of charge distribution. However, the decoherence effect we compute from ion-ion collisions and distant ion interactions are so strong that we can neglect any other decoherence source. Moreover, we will see that the decoherence time does not strongly depend on the different concentrations of the different ionic solutions mentioned above.

The density matrix for the single ion may be shown to be proportional to a function $f\left(\mathbf{x}, \mathbf{x}^{\prime}, t\right)$ which does not depend on the ion state [16, 11. Here $\mathbf{x}$ denotes the ion position. For scattering of environment particles with de Broglie wavelength $\lambda$, we have

$$
f\left(\mathbf{x}, \mathbf{x}^{\prime}, t\right)=e^{-\Lambda t\left(1-e^{-\frac{\left|\mathbf{x}^{\prime}-\mathbf{x}\right|^{2}}{2 \lambda^{2}}}\right)},
$$


that is,

$$
f\left(\mathbf{x}, \mathbf{x}^{\prime}, t\right) \sim\left\{\begin{array}{ll}
e^{-\Lambda t \frac{\left|x^{\prime}-x\right|^{2}}{2 \lambda^{2}}} & \text { for }\left|\mathbf{x}^{\prime}-\mathbf{x}\right| \ll \lambda \\
e^{-\Lambda t} & \text { for }\left|\mathbf{x}^{\prime}-\mathbf{x}\right| \gg \lambda
\end{array},\right.
$$

where $\Lambda \equiv n\langle\sigma v\rangle$ is the scattering rate cross section. The product of the cross section $\sigma$ by the velocity $v$ is averaged over the thermal velocity distribution at $T=310 K, n$ is the density of the scatterer centers (ions). Eq. (3) implies that for the single ion the spatial superposition decays exponentially as $\Lambda^{-1}$ and that for $N$ ions as $(\Lambda N)^{-1}$. Let us consider the case of $\mathrm{Na}^{+}$and $\mathrm{Cl}^{-}$ions solution. The de Broglie wavelength of the $\mathrm{Na}^{+}$ion (mass $m_{\mathrm{Na}}=22.990 \mathrm{amu}$ ) or the $\mathrm{Cl}^{-}$ion (mass $\left.m_{C l}=35.453 \mathrm{amu}\right)$ is

$$
\lambda=\frac{2 \pi \hbar}{\sqrt{3 m k T}} \sim 0.3 \AA .
$$

In the present case of high ionic concentration, the smallest acceptable inter-ionic distance $\left(\left|\mathbf{x}-\mathbf{x}^{\prime}\right|\right)$ cannot be of course smaller than the one corresponding to the elementary cell of the crystalline salt to be formed, which typically is of the order of few $\AA$. Even for such a distance we are clearly in the $\left|\mathbf{x}-\mathbf{x}^{\prime}\right| \gg \lambda$ limit of equation (2). (Such a condition is certainly satisfied also in the case of the other, smaller concentrations of the other considered ionic solutions). In the case of two ion-ion collision with unit charge $q_{e}$ and with relative velocity $v$ the cross section is evaluated to be [16]

$$
\sigma \sim\left(\frac{g q_{e}^{2}}{m v^{2}}\right)^{2}
$$

where $g=1 / 4 \pi \epsilon_{0}$ is the Coulomb constant. The decoherence time $\tau_{1} \equiv(\Lambda N)^{-1}$ for $N$ ions is then given by

$$
\tau_{1} \sim \frac{\sqrt{m(k T)^{3}}}{N n g^{2} q_{e}^{4}}
$$

We have used $v \sim \sqrt{\frac{k T}{m}}$ at thermal equilibrium. $N$ is taken to be of the order of $10^{23}$ (about a mole of ions), and for $\mathrm{Na}^{+}$and $\mathrm{Cl}^{-}$at the crystallization point, it is $n=2.163 \times 10^{3} \mathrm{~kg} \mathrm{~m}^{-3}$ [17]. Then we have:

$$
\tau_{1} \sim \frac{\sqrt{m(k T)^{3}}}{10^{23} \frac{2163}{m_{N a}+m_{C l}} g^{2} q_{e}^{4}} \sim 4.610^{-40} \mathrm{~s} .
$$

The order of magnitude of this result does not change much even for the other ionic solutions, which confirms the correcteness of the used approximations. The $\tau_{1}$ values we obtain for the other ionic solutions are reported in Table 1.

In a similar way, one can show that the decoherence time $\tau_{2}$ for the interaction with distant ions is given by [16]:

$$
\tau_{2} \sim \frac{\sqrt{m(k T)}}{N n a g q_{e}^{2}} .
$$


In this case the smallest acceptable distance between two $\mathrm{Na}^{+}$ions is $a=5.64 \AA$ [17] (the edge of the cubic elementary cell).

In the following table we report the decoherence time for ion-ion collisions $\left(\tau_{1}\right)$ and for interactions with distant ions $\left(\tau_{2}\right)$ in the ionic solutions for the formation of a set of crystalline binary compounds.

Table 1. Decoherence time for ion-ion collisions $\left(\tau_{1}\right)$ and for interactions with distant ions $\left(\tau_{2}\right)$

\begin{tabular}{crr}
\hline \hline salts & $\tau_{1} / 10^{-40} s$ & $\tau_{2} / 10^{-38} s$ \\
\hline $\mathrm{NaF}$ & 2.6 & 4.9 \\
$\mathrm{NaCl}$ & 4.6 & 4.4 \\
$\mathrm{NaBr}$ & 5.5 & 4.9 \\
$\mathrm{NaI}$ & 7.1 & 5.8 \\
$\mathrm{KF}$ & 5.1 & 5.2 \\
$\mathrm{KCl}$ & 8.3 & 7.1 \\
$\mathrm{KBr}$ & 9.6 & 7.9 \\
$\mathrm{CsF}$ & 13.4 & 12.0 \\
$\mathrm{CsCl}$ & 17.3 & 21.0 \\
$\mathrm{CsBr}$ & 19.6 & 25.6 \\
$\mathrm{CsI}$ & 23.6 & 27.8 \\
$\mathrm{AgCl}$ & 9.5 & 9.2 \\
$\mathrm{AgBr}$ & 10.7 & 10.0 \\
$\mathrm{AgI}$ & 15.3 & 12.7 \\
$\mathrm{ZnS}$ & 7.2 & 7.2 \\
$\mathrm{PbS}$ & 16.2 & 14.7 \\
\hline \hline
\end{tabular}

According to Table 1, due to the very short decoherence time, there would be no possibility for the formation of the considered crystals to occur, which of course contradicts the common experience (in practice the crystal formation lasts for a time many orders of magnitude longer than $\tau_{1}$ and $\tau_{2}$ in Table 1 ). We note that in the computation of the decoherence time we have used for the ions in the solution the shortest possible inter-ionic distances (and therefore the most favorable to avoid decoherence). We also remark, that in the case in which the crystal is described as a quantum mechanical n-body system, the wave functions of the constituent ions are centered at the proper lattice sites and present spatial superposition over distances of the order of the lattice length. Then one could be tempted to conclude that the above results could apply to the ions which form the crystals. This conclusion is, however, clearly wrong. The crystal would not even be stable in this case, in evident contradiction with common experience. 
The way out of these contradictions is in the fact that the QM description is not completely adequate for the description of the process of the crystal formation and for the crystal system: as said in the introductory remarks, the crystal phase transition process and the crystal system are many-body QFT problems and the binding of the atoms in the crystalline lattice is due to the long range correlation mediated by the NG bosons (the QFT formalism can be found in full length and details in standard textbooks, see e.g. [8]). In the present case, the decoherence mechanism thus points to the borderline between QM and QFT.

The inter-ionic distances used in our computations are of course comparable with the wavelength used in the $X$-ray diffraction experiments with the systems we consider, which is $\lambda \sim 1.5 \AA$. This corresponds to oscillation time $\tau_{X}$ of the order of $0.5 \times 10^{-18} s$, much longer than $\tau_{1,2}$ in Table 1 . Suppose, however, one wants to insist in using, e.g., Eq. (5). Then, we have

$$
\frac{\tau_{1}}{\tau_{X}}=\frac{n_{X}}{n}
$$

i.e. the $X$-ray typical diffraction time $\tau_{X}$ would correspond to an extremely diluted solution where the density $n_{X}$ of the scatterer centers, say $\mathrm{Na}$ and $\mathrm{Cl}$ ions, is of the unacceptable order of $10^{-18}$, and, from

$$
n_{X}=10^{-18}=\frac{\left(m_{N a}+m_{C l}\right) a m u}{a^{3}}
$$

we have the equally absurd ion spacing of the order of $a \sim 10^{-3} \mathrm{~m}=10^{7} \stackrel{\circ}{A}$. Equally unacceptable conclusions are reached by using Eq. (7).

Again we see that in the case here discussed the QM decoherence picture and formulas cannot be used. They are not consistent with familiar experimental methodologies. Since these methodologies, on the other hand, confirm the quantum dynamical nature of the system, the inconsistency points to the limit of applicability of QM in favor of QFT. As far as we know, the existing literature has not paid attention to this side of the confining limits of QM by using the decoherence phenomenon.

In conclusion, according to our discussion, decoherence may be then promoted to the relevance of a criterion, able to discriminate between QM and QFT, from one side, and classical mechanics from the other side. Thus, generally speaking, and contrarily to current common belief, decoherence does not necessarily signals the approaching to the classical mechanics regime; it may also signals the approaching to the QFT regime, indeed. One must carefully consider the physics of the system under study in order to correctly conclude on the implications of decoherence.

This work has been partially supported by INFN, by MURST, by INFM and by an ESF Network on Topological Defects. 


\section{Bibliography}

[1] H.D. Zeh, Found. Phys. 169 (1970)

[2] W.H.Zurek, Phys. Rev. D 241516 (1981)

[3] W.H.Zurek, Phys. Rev. D 261862 (1982)

[4] E.Joos and H.D.Zhe, Z. Phys. B 59223 (1985)

[5] D.Giulini, E.Joss, C.Kiefer, J.Kupsch, I.O.Stamatescu and H.D.Zeh, Decoherence and the appearance of a classical world in quantum theory (Springer, Berlin, 1996)

[6] C. Itzykson and J.B. Zuber, Quantum field theory, (McGraw-Hill Inc., 1980)

[7] P.W. Anderson, Basic Notions of Condensed Matter Physics, (Menlo Park, Benjamin, 1984)

[8] H. Umezawa, H. Matsumoto and M. Tachiki, Thermo Field Dynamics and Condensed States (North-Holland Pub. Co., Amsterdam 1982)

[9] H. Umezawa, Advanced Field Theory: Micro, Macro, and Thermal Concepts (American Institute of Physics, N.Y., 1993)

[10] N.N. Bogolubov, A.A. Logunov and I.T. Todorov Introduction to Axiomatic Quantum Field Theory, (W.A. Benjamin, Reading, MA, 1975)

[11] G. L. Sewell, Quantum Theory of Collective Phenomena, (Oxford Clarendon Press, 1986)

[12] O. Bratteli and D. W. Robinson, Operator Algebras and Quantum Statistical Mechanics (Springer, Berlin, 1979)

[13] H.Umezawa and G.Vitiello, Quantum Mechanics (Bibliopolis, Napoli, 1985)

[14] G.Rickayzen, Theory of superconductivity (John Wiley and Sons, New York, 1965)

[15] Kuster-Thiel, Tabelle per le analisi chimiche e chimico-fisiche (Hoepli, Milano 1988) 
[16] M.Tegmark, Found. Phys. Lett 6571 (1993); Phys. Rev. E 614194 (2000)

[17] Powder Diffraction File 2.02, release 1999 